\title{
Investigation of Common Bacterial Pathogens Leading to Urinary Tract Infections Among Patients with Diabetes Mellitus in Sub-Saharan Africa.
}

\author{
Jackline Nakkungu ${ }^{a}$ \\ a Department of Microbiology, Faculty of Health Sciences, University of Kisubi, \\ Kisubi, Uganda
}

Abstract

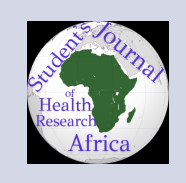

Background: ${ }^{a}$

The risk of diabetic patients acquiring UTIs is higher than that of their non-diabetic counterparts. This is due to the damage on the urinary bladder nerves caused by high blood glucose levels. This nerve damage reduces the ability of the urinary bladder to sense the presence of urine in it. As a result, urine stays for a long time in the bladder, increasing the growth of the bacteria in urine.

UTI's may lead to emphysematous pyelonephritis, cystitis in females, renal and perirenal abscess, papillary necrosis, xanthogranulomatous pyelonephritis, and fungal infections. There is limited knowledge about the bacteria affecting diabetic patients in Uganda.

Methodology:

In a cross-sectional study, 76 diabetic patients at Our Lady of Consolata Kisubi Hospital had their urine samples collected and analyzed for the presence of uro-bacterial pathogens in the microbiology laboratory at the University of Kisubi. Patients were instructed on how to collect midstream urine, which were cultured on blood agar and MacConkey agar plates. The bacteria were identified according to colony size, shape, and hemolysis. Several biochemical tests were used to confirm the presence of the different bacteria strains from the individual subcultured colonies

Results

Escherichia coli was the most predominant bacterium associated with UTIs in diabetes mellitus with an abundance of $42.3 \%$. This was followed by Klebsiella species with $21.1 \%$, Staphylococcus aureus with $14.1 \%$, Streptococcus species at $11.76 \%$, Proteus species at 5.8\%, Enterococcus faecalis at 3.5\%, and Serratia marcescens with $1.17 \%$.

Female diabetic patients were found to be more infected with uropathogenic than their male diabetic counterparts. Conclusion and recommendations:

Escherichia coli, Klebsiella species, Staphylococcus aureus, Streptococcus species, Proteus species, Serratia marcescens, and Enterococcus faecalis were identified to be commonly found in the urine of patients with diabetes mellitus. More screening and early management of such infections is needed in Sub-Saharan Africa.

\footnotetext{
${ }^{a}$ email: jackieerics@gmail.com date recieved: 9th/02/2021 date accepted: 10th/03/2021
} 


\section{Background}

In 2014, Lastours reported that "Diabetic patients have a higher incidence of UTI than their nondiabetic counterparts" (Lastours et al., 2014). And that a severe UTI can be a cause of complications, ranging from dysuria to organ damage and sometimes death. UTI's are therefore a serious health threat to diabetic patients.

The World Health Organization also estimated that globally, over 365 million diabetic patients have UTIS (WHO, 2013). They also found that UTIs often results in an increased death rate among diabetic patients. In 2011 for example, 1.2 million deaths among diabetic patients were recorded to be due to UTIs (WHO, 2013).

Patients with type 2 diabetes mellitus are at an increased risk for UTIs. This is due to incomplete urinary bladder emptying caused by autonomic neuropathy. This nerve damage allows urine to stay for long in the bladder which favors the growth of bacteria (Truzzi et al., 2008: Johnson et al., 2013). Other factors that were found to enhance the risk for UTI in diabetics include age, metabolic control, and long-term complications, primarily diabetic nephropathy and cystopathy (Nitzan, 2015).

Average medical expenditures for diabetics are 2.3 times higher than non-diabetics, adjusting for differences in both age and sex. Progression of diabetes is associated with a variety of microand macrovascular events, including blindness, nephropathy, stroke, and heart disease.

The increased risk of UTI among diabetic patients, coupled with the increase in the incidence of type 2 diabetes mellitus worldwide in recent years, may impose a substantial burden on medical costs (Nitzan et al., 2015). Also, the high rates of antibiotic prescription, including broad-spectrum antibiotics, for UTI in these patients may further induce the development of antibiotic-resistant urinary pathogens (Nitzan, 2015).

The risk of diabetic patients acquiring UTIs is higher than that of their non-diabetic counterparts. This is due to the damage on the urinary bladder nerves caused by high blood glucose levels. This nerve damage reduces the ability of the urinary bladder to sense the presence of urine in it. As a result, urine stays for a long time in the bladder, increasing the growth of the bacteria in urine (Johnson et al., 2013).
UTI's may lead to emphysematous pyelonephritis, cystitis in females, renal and perirenal abscess, papillary necrosis, xanthogranulomatous pyelonephritis, and fungal infections.

This research, therefore, intends to identify the common bacterial pathogens leading to UTIs in diabetic patients. This can help in the management of UTIs and their associated complications in diabetic patients.

\section{METHODOLOGY}

\section{Study design}

This was a cross-sectional study where diabetic patients who consented to take part in the study were to have their urine samples collected and analyzed for the presence of bacterial pathogens.

\section{Study site}

Samples were collected from diabetic patients at Our Lady of Consolata Kisubi Hospital and immediately transported to the microbiology laboratory at the University of Kisubi for analysis.

\section{Sample population}

Sample size was estimated using the formula $\mathrm{n}=4 \mathrm{pq} / \mathrm{L2}$ (Martin 1988).

Where:

$\mathrm{n}=$ sample size required

$p=$ estimated number of diabetic patients of attending $q=1-p$ kisubi hospital, diabetes clinic.

$\mathrm{L}=$ desired errors (required precision).

$p=74.5 \%$

Desired error of $10 \%(0.1)$,

$$
\begin{aligned}
& \mathrm{n}=4 \times 0.745(1-0.745) \\
& 0.1^{2}
\end{aligned}
$$$$
\mathrm{n}=76
$$

Thus a sample size of 76 was used.

\subsection{Exclusion criteria and Inclusion criteria}

\section{Inclusion criteria}

Diabetic patients aged 18 years and above attending the diabetic clinic at kisubi hospital, and consented to participate in the study.

\section{Exclusion criteria}

Diabetic patients below the age of 18 years.

\section{Sample collection and processing}

Upon consenting to take part in the study, participants were instructed by the researcher on how to 
appropriately collect a midstream urine sample to avoid contamination.

Urine samples were immediately transported in a cool box to the microbiology laboratory for examination. All urine samples were inoculated using a sterile wire loop on the different types of media i.e. blood agar and MacConkey plates and streak to obtain single colonies and stained to identify gram-negative and gram-positive organisms and to identify bacteria by cell morphology. All plates were incubated at 37 degrees Celcius for 24 to 48 hours for visible growth.

All processes were done using standard operating procedures (SOPs).

Materials
MacConkey agar
Blood agar
Distilled water
Incubator
Heat source
Microbiology plates
Sterile urine sample bottles
Inoculation wire loops/inoculation wires
Microscope slides
Microscope
Autoclave
Gram stains
Centrifuge

\section{Collection of urine samples}

Patients were instructed on how to collect midstream urine, below is the procedure on how it was collected.

The patient was given a sterile dry wide-necked leak-proof container and requested to collect 10 to $20 \mathrm{mls}$ of urine.

Females were asked to wash their hands and cleanse the area around the urethral opening with clean water, dry the vagina with a sterile gauze pad and then collect the urine with the labia held apart. They were asked to urinate the first portion of urine in the toilet, and then trap the midstream urine into the sterile urine container and the remaining urine to be passed out in the toilet.

Males were also asked to wash their hands then collect the midstream urine.

The container was then labeled with the date, laboratory identification number of the patient, patient's name, and age took for examination.

\section{Culture of urine specimen.}

Urine specimens were cultured on blood agar and MacConkey agar plates. The plates were la- beled with the patient identification number and date of culture.

The urine sample was homogenized, and then a sterile wire loop will be held vertically and immersed into the sample to collect a loop full of urine for the first inoculum.

\section{Inoculation technique}

Urine samples were cultured on both blood agar and MacConkey agar plates. Both plates were incubated at 350-370 C in an electrothermal incubator for18-24 hours.

\section{Reading of plates}

Table 1: Quantification of colonies

\section{Identification of bacteria}

Identification of bacteria on blood agar and MacConkey agar

After 24 hours, the plates were inspected for bacterial growth. The bacteria were identified according to colony size, shape, and hemolysis. On MacConkey agar, only gram-negative bacteria grew and were either lactose fermenters or nonlactose fermenters. The suspected colonies were further subcultured on Nutrient agar to obtain pure colonies that were confirmed using biochemical tests. Colonies formed on different plates were gram stained for different gram-positive and gramnegative rods or cocci.

\section{Gram staining}

Gram staining was performed on colonies found on blood agar to describe the morphology of bacteria and to classify them as either gram-positive or gram-negative

\section{Confirmatory tests for the bacteria strains}

Several biochemical tests were used to confirm the presence of the different bacteria strains from the individual subcultured colonies. These are discussed below.

\section{Triple sugar iron test \\ Principle}

TSI agar contains three sugars, glucose, sucrose, and lactose, and also ferrous iron (iii) sulphate. Also, it has an indicator phenol red that changes color according to the $\mathrm{pH}$ change. For the nonlactose fermenters, they breakdown the glucoseproducing acid that lowers the $\mathrm{pH}$ turning the indicator to yellow in the butt. After the breakdown of the glucose these organisms now breakdown the amino acids present in the medium raising the $\mathrm{pH}$ that turns the indicator red in the slant. For the 
Table 1. Quantification of colonies

\begin{tabular}{ll}
\hline Number of colonies on plate & Colony forming units \\
$1-9$ & $10^{2}$ \\
$10-99$ & $10^{3}$ \\
100 & $10^{4}$ \\
$>100$ & $10^{5}$ \\
\hline
\end{tabular}

Table 2. Interpretation of colony counts of urine

\begin{tabular}{|c|c|c|}
\hline $\begin{array}{l}\text { Detected } \\
\text { growth }\end{array}$ & Midstream urine & In and out catheter urine. \\
\hline $\begin{array}{l}1 \text { type of } \\
\text { organism }\end{array}$ & $\begin{array}{l}1-9 \text { colonies (not significant) } 104 \mathrm{CFU} / \mathrm{ml} \text { (growth is } \\
\text { significant) }\end{array}$ & $\begin{array}{l}\text { 1-9 colonies(not significant) } 104 \\
\text { CFU/ml(growth is significant) }\end{array}$ \\
\hline $\begin{array}{l}2 \text { types of } \\
\text { organisms }\end{array}$ & Each>105 CFU/ml(growth is significant) & Each $>105$ CFU $/ \mathrm{ml}$ (growth is significant) \\
\hline $\begin{array}{l}>2 \text { types of } \\
\text { organisms }\end{array}$ & $\begin{array}{l}\text { Mixed growth, probably due to contamination. } \\
\text { Request a repeat sample collection. }\end{array}$ & $\begin{array}{l}\text { If one isolate was dominant } \\
>104 \text { CFU/ml (growth is significant) }\end{array}$ \\
\hline
\end{tabular}

lactose fermenters, they break down all the three sugars producing enough acid to turn the color of the indicator in the butt and the slant to yellow. The hydrogen sulphide produced by some organisms reacts with iron (iii) sulphate that turns the media black.

\section{Procedure}

TSI tubes were inoculated with a long straight sterile wire. The well-isolated test colony recovered from the agar plate was touched with the end of the inoculating needle which was then stabbed into the deep of the tube. When the inoculating wire was removed from the deep of the tube, the slant surface was streaked with a back and forth motion.

Inoculated tubes were placed into the incubator at $350 \mathrm{C}$ for 18 to 24 hours.

\section{Citrate Utilization test \\ Principle}

This test is one of several techniques used to assist in the identification of enterobacteria. The test is based on the ability of an organism to use citrate as the only source of carbon and ammonia as its only source of nitrogen. In the presence of citrate utilizing microbe, sodium citrate that is contained in the medium is broken down and this results in a change in $\mathrm{pH}$ that causes the color of the indicator to change to blue.

\section{Procedure}

The agar was inoculated with a single colony from the subculture using a long straight wire. The well-isolated test colony recovered from a gar plate was touched with the end of the inoculating needle which was then stabbed into the deep of the tube. When the inoculating wire was removed from the deep of the tube, the slant surface was streaked with a back and forth motion. Inoculated tubes were placed into the incubator at $350 \mathrm{C}$ for 18 to 24 hours

\section{SIM test (Sulphur indole motility)}

SIM medium is a semisolid a gar used to determine hydrogen sulphide production, indole formation, and motility.

SIM medium is used to differentiate members of the family Enterobacteriaceae. Haziness that spreads from the stab line will indicate a positive test for motility.

A red color development after the addition of Kovacs reagents will indicate indole production. A black precipitate will indicate hydrogen sulphide production.

\section{Urease test}

The Urease test identifies those organisms that are capable of hydrolyzing urea to produce ammonia and carbon dioxide. It is primarily used to distinguish urease-positive protease from other Enterobacteriaceae.

\section{Principle}

Many organisms especially

those that infest the urinary

tract has a urease enzyme which is 
able to split urea in the presence of water to release

ammonia and carbon dioxide. The ammonium carbonate which turns the medium alkaline

to form the indicator phenol

red from its original orange-yellow

color to bright pink. No color change will be observed in the absence of urease-producing bacteria.

\section{Procedure for urease test}

Using a sterile wire loop, a single colony of the bacteria was streaked on the surface of the urea agar slant. The tube was left loosely capped to allow entry of oxygen. The tube was then incubated for 48 hours to 7 days at 35 degrees Celcius.

The color of the medium changed from light orange to magenta if the organism produced the enzyme urease.

\section{Quality control}

Known organisms from the ATCC plates were used for proper identification of organisms. All procedures were to take place in a sterile environment to prevent contamination.

\section{Data analysis, interpretation, and management}

Numbers of the different identified bacterial pathogens were recorded in a census table and their respective prevalences were determined using percentages. A pie chart was used to compare the prevalence of these pathogens.

\section{Results}

A total of 76 diabetic patients with or without symptoms of UTIs were examined. The participants were ranging from $35-85$ years of age, with a mean age of 64.6 years. Of the 76 participants, 6 were found not to have significant bacterial growth while 70 were infected of whom some had a mixed infection.

Figure 1: The figure is a pie chart showing the prevalence of the uropathogens in patients of diabetes mellitus.

From the pie chart above, it is implied that out of the 85 isolates, 60 were Gram negative while 25 were Gram positive bacteria. Escherichia coli was the most prevalent bacterium with an abundance of $42.3 \%$. This was followed by Klebsiella spieces with $21.1 \%$ abundance, then Staphylococcus aureus with $14.1 \%$, Streptococcus spieces at $11.76 \%$, Proteus spieces at 5.8\%, Enterococcus faecalis at 3.5\% and the list abundant being Serratia marcescens with $1.17 \%$.

Figure 2: The figure is a bar-graph showing the distribution of uropathogens in patients of diabetes mellitus by gender.

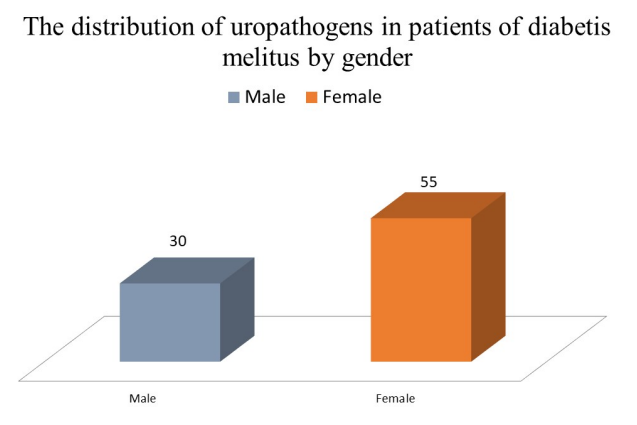

From the bar graph above, it is illustrated that female diabetic patients were more infested with uropathogens than their male diabetic counterparts. Out of the 85 bacterial isolates, 55 were from female participants compared to the 30 isolated from the male participants, so females were more infected than males.

\section{Discussion}

This study focused on investigating bacterial pathogens leading to urinary tract infections in patients with diabetes mellitus including identifying the most common uropathogens, determining their prevalence and their distribution by gender in the study population.

A total of 85 isolates were recovered from 76 samples indicating mixed infections in some patients. Six (6) samples did not have significant growth, so isolates discussed in this study came from 70 samples.

This study showed a prevalence of $92.1 \%$ uropathogens among patients with diabetes mellitus. This finding is not very surprising because it is known that the high glucose concentration in the urine of patients with diabetes mellitus may create a culture medium for pathogenic bacteria that may even lead to immunologic impairment ( Peters, et al., 2012).

Escherichia coli was the most prevalent uropathogen with $42.3 \%$. This finding is within the range of the other studies like the $47 \%$ reported in the Netherlands, the 56\% reported in India, 
Table 3. The table summarizes the distribution of the different strains of uropathogens by gender isolated from 70 participants.

\begin{tabular}{llll}
\hline UROPATHOGENS & MALE & FEMALE & TOTAL \\
Enterococcus faecalis & 1 & 2 & $\mathbf{3}$ \\
Staphylococcus aureus & 4 & 8 & $\mathbf{1 2}$ \\
Streptococcus spieces & 4 & 6 & $\mathbf{1 0}$ \\
Escherichia coli & 12 & 24 & $\mathbf{3 6}$ \\
Proteus spieces & 2 & 3 & $\mathbf{5}$ \\
Klebisella species & 7 & 11 & $\mathbf{1 8}$ \\
Serratia marcescens & 0 & 1 & $\mathbf{1}$ \\
TOTAL & $\mathbf{3 0}$ & $\mathbf{5 5}$ & $\mathbf{8 5}$ \\
\hline
\end{tabular}

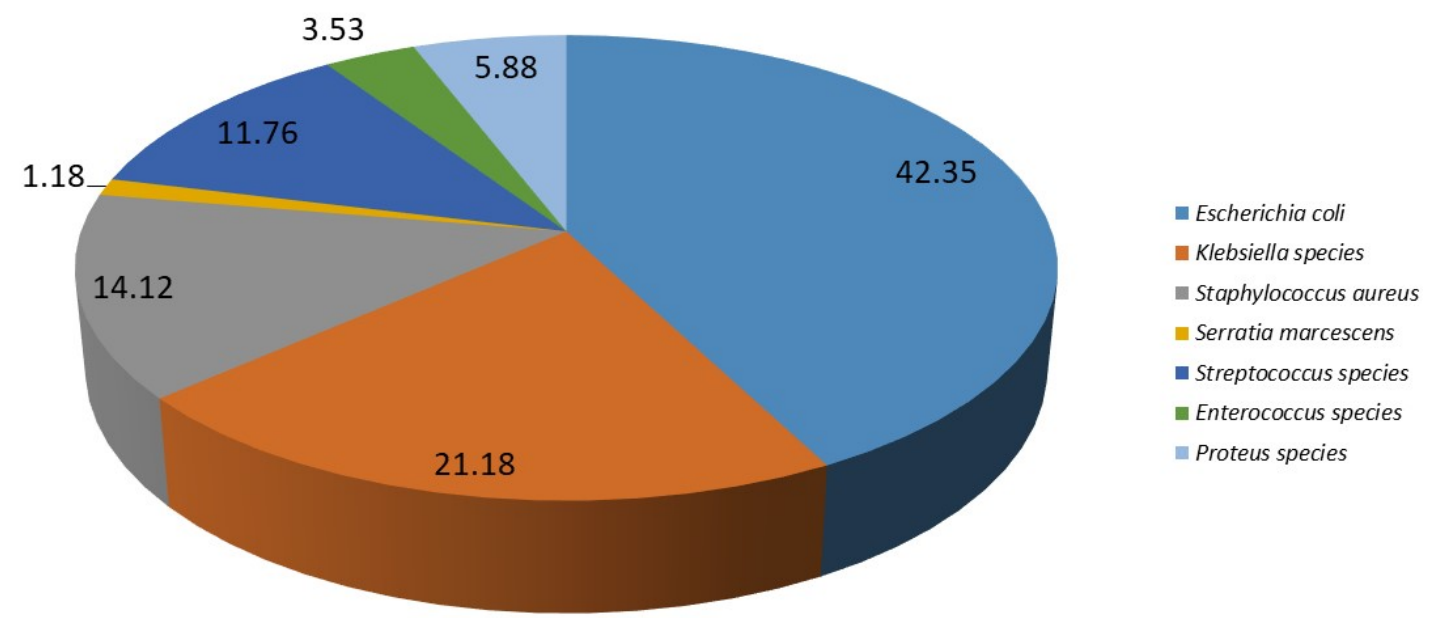

Chart 1. The figure is a pie chart showing the prevalence of the uropathogens in patients of diabetes mellitus.

and the $41.5 \%$ reported in Yemen. (Ampaire et al., 2015). Klebsiella pneumonia came in second place with $21.1 \%$. In general, the findings confirm that the types of uropathogens present in diabetic patients and their relative abundance is relatively the same.

\section{Conclusions and Recommendations}

Seven different species of bacteria were identified to be commonly found in the urine of patients with diabetes mellitus. These included: Escherichia coli, Klebsiella species, Staphylococcus aureus, Strep- tococcus species, Proteus species, Serratia marcescens, and Enterococcus faecalis.

Escherichia coli was the most predominant bacterium associated with UTIs in diabetes mellitus with an abundance of $42.3 \%$. This was followed by Klebsiella species with $21.1 \%$ abundance, then Staphylococcus aureus with $14.1 \%$, Streptococcus species at $11.76 \%$, Proteus species at $5.8 \%$, Enterococcus faecalis at $3.5 \%$ and the least abundant being Serratia marcescens with $1.17 \%$.

Female diabetic patients were found to be more infested with uropathogens than their male diabetic counterparts. Out of the 85 bacterial isolates, 55 were from female participants compared to the 
30 isolated from the male participants, so females were more infected than males.

I recommend that a more reliable technique of bacterial identification such as DNA determination by PCR should be used to be sure of having more reliable findings. The science of biochemical tests is less specific with relatively low sensitivity.

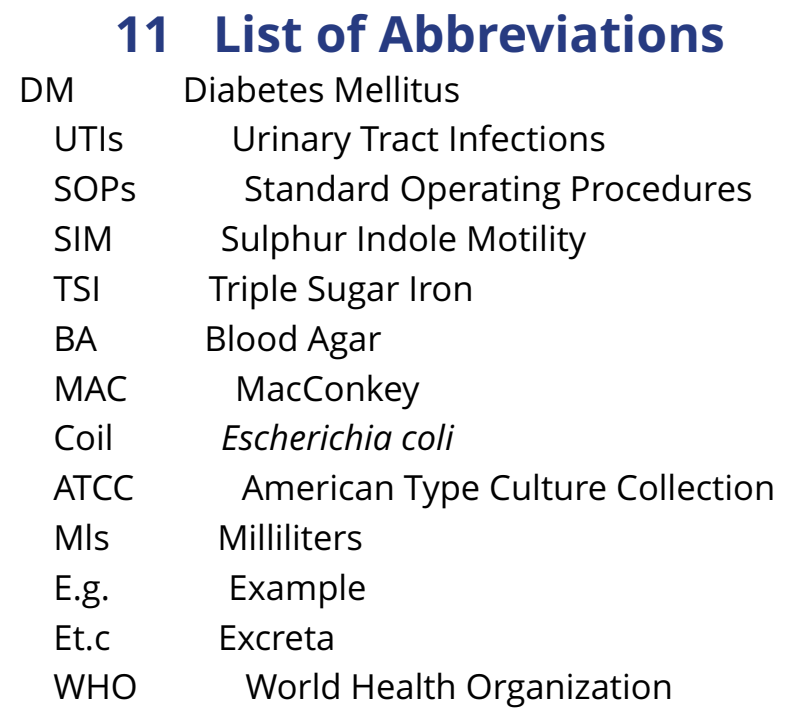

\section{Definition of terms}

Emphysematous pyelonephritis: It is a severe, necrotizing infection of the renal parenchyma; it causes gas formation within the collecting system and perirenal tissues.

Cystitis: Inflammation of the urinary bladder.

Papillary necrosis: Disorder of the kidneys on which all or part of the renal papillae die.

Abscess: A swollen area within body tissue, containing an accumulation of pus.

Xanthogranulomatous pyelonephritis: A chronic inflammatory disorder of the kidney characterized by a destructive mass that invades the renal parenchyma.

Uropathogens: These are bacteria pathogens found in urine. 
Table 4. References

1) Ampaire, L., Butoto, A., Orikiriza, P., \& Muhwezi, O. (2015). Bacterial and Drug Susceptibility Profiles of Urinary Tract Infection in Diabetes Mellitus Patients at Mbarara Regional Referral Hospital, Uganda. British Microbiology Research Journal, 9(4), 1-5.https://doi.org/10.9734/BMRJ/2015/17483

2) de Lastours, V., \& Foxman, B. (2014). urinary tract infection in diabetes: epidemiologic considerations, Current Infectious Disease Reports. Vol.16, no.1 article 389https://doi.org/10.1007/s11908-013-0389-2

3) Johnson, K., A. Ptaszynska, A., Schmitz, B., Sugg, J., Parikh, S., \& List, J. (2013). urinary tract infections in patients diabetes treated with dapagliflozin, Vol.27 ,no.5,pp473-478. Journal of diabetes and its complicatio ns.https://doi.org/10.1016/j.jdiacomp.2013.05.004

4) Nitzan, O., Elias, M., Chazan, B., \& Saliba, W. (2015). Urinary tract infections in patients with type 2 diabetes mellitus: review of prevalence, diagnosis, and management. Diabetes, metabolic syndrome and obesity : targets and therapy, 8, 129-136.https://doi.org/10.2147/DMSO.S51792

5) Peters, B. M., Jabra-Rizk, M. A., Graeme, O., Costerton, J. W., \& Shirtliff, M. E. (2012). Polymicrobial interactions: impact on pathogenesis and human disease. Clinical microbiology reviews, 25(1), 193-213.http s://doi.org/10.1128/CMR.00013-11

6) Truzzi J, C., Almeida F, M., Nunes E, G., \& Sadi M, V. (2008). Residual urinary volume and urinary tract infection. J Urol.https://doi.org/10.1016/j.juro.2008.03.044

7) World Health Organization Global Report on Diabetes Geneva accessed August . (2013) 